\title{
Overview of Secondary Neurulation
}

\author{
Martin Catala \\ Laboratoire de Biologie du développement, Sorbonne Université, Paris, France
}

Secondary neurulation is a morphological process described since the second half of the 19th century; it accounts for the formation of the caudal spinal cord in mammals including humans. A similar process takes place in birds. This form of neurulation is caused by the growth of the tail bud region, the most caudal axial region of the embryo. Experimental work in different animal species leads to questioning dogmas widely disseminated in the medical literature. Thus, it is clearly established that the tail bud is not a mass of undifferentiated pluripotent cells but is made up of a juxtaposition of territories whose fate is different. The lumens of the two tubes generated by the two modes of neurulation are continuous. There seem to be multiple cavities in the human embryo, but discrepancies exist according to the authors. Finally, the tissues that generate the secondary neural tube are initially located in the most superficial layer of the embryo. These cells must undergo internalization to generate the secondary neurectoderm. A defect in internalization could lead to an open neural tube defect that contradicts the dogma that a secondary neurulation defect is closed by definition.

Key Words : Secondary neurulation · Tail bud · Cavitation · Blastema · Caudal spinal cord.

\section{INTRODUCTION}

The first stages of nervous system formation take place during the 3rd and 4th weeks of development (weeks after fertilization) in humans. The first event that marks the initiation of this development is neural induction, a major phenomenon that transforms the primary ectoderm (the most superficial layer of the embryo) into neurectoderm and surface ectoderm. Neurectoderm forms the neural plate that will generate the central nervous system and the vast majority of the peripheral nervous system. Surface ectoderm, for its part, generates the epidermis, it also participates in the formation of some elements of the cephalic peripheral nervous system as well as in the genesis of the oral and nasal epithelium.

Neurulation is the series of morphogenetic phases that lead to the transformation of this neural plate into a neural tube. In humans, several processes account for the formation of the neural tube. Primary neurulation is the most classic one, it leads to a change in the shape of the neural plate, the lateral edges of which rise up and then meet on the dorsal midline to merge. This phenomenon is well known to physicians and surgeons. However, it should be noted that there are anatomical variations depending on the position along the anteroposterior (rostro-caudal) axis. Primary neurulation ends with the closure of the neuropores (rostral and caudal). After closure of the caudal neuropore, the spinal cord is not completely

- Received : December 24, 2020 •Revised : December 30, 2020 •Accepted : January 5, 2021

- Address for reprints : Martin Catala

Laboratoire de Biologie du développement, Sorbonne Université, 9 Quai Saint Bernard, Paris 75005, France

Tel : +33-142161316, Fax : +33-142161321, E-mail : martin.catala@sorbonne-universite.fr, ORCID : https://orcid.org/0000-0003-1554-9007

This is an Open Access article distributed under the terms of the Creative Commons Attribution Non-Commercial License (http://creativecommons.org/licenses/by-nc/4.0) which permits unrestricted non-commercial use, distribution, and reproduction in any medium, provided the original work is properly cited. 
generated and the most caudal part of this anatomical region is produced by a different mechanism. Since this morphogenetic process occurs after the one affecting the neural plate, it is described as secondary neurulation. I will be interested here in this phase of morphogenesis.

\section{MORPHOLOGICAL ASPECT OF SECONDARY NEURULATION}

\section{First historical observations of secondary neuru- lation}

Emil Gasser (1847-1919) described in 1879 chick and goose embryos in both sagittal and axial sections at different stages

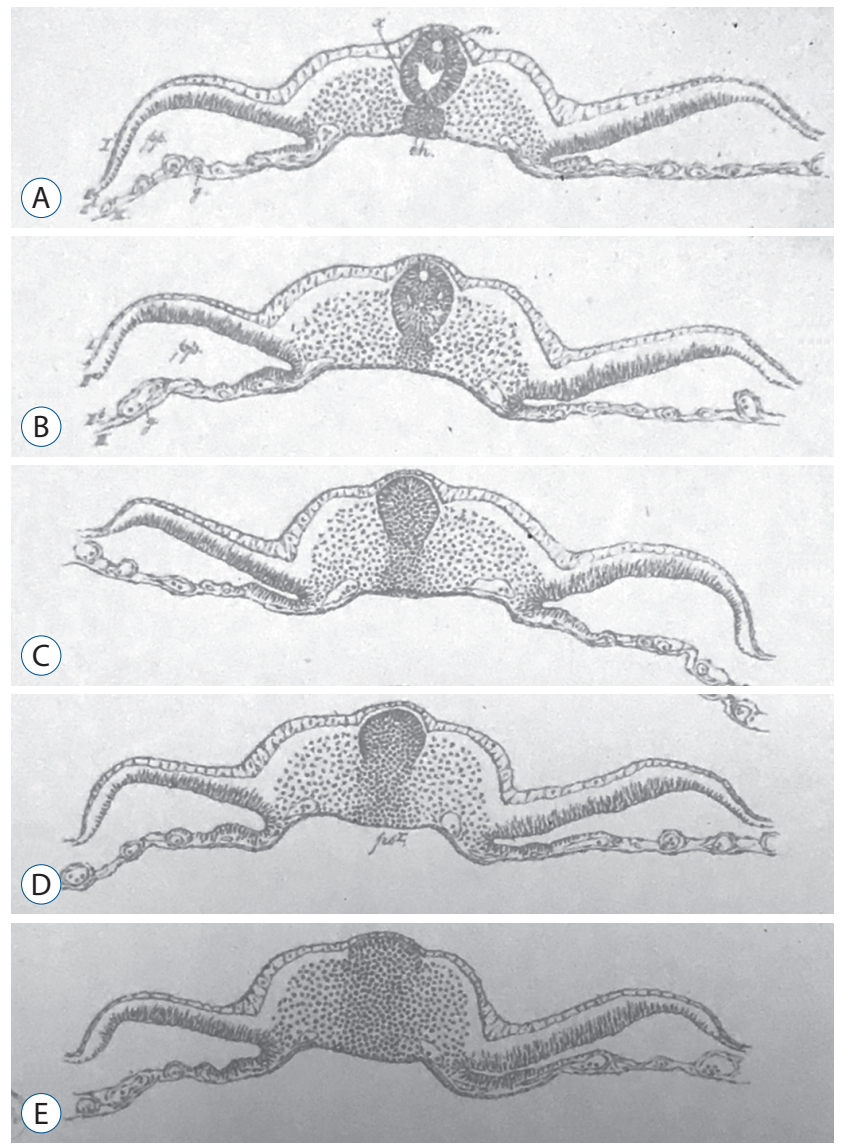

Fig. 1. Figures from plate 5 published by Gasser ${ }^{11)}$ showing axial sections of chick embryo from rostral (A) to caudal (E). This plate presents the first historical illustration of secondary neurulation in chick embryos. Multiple lumens can be evidenced in the secondary neural tube (A and B). C: The primordium of the spinal cord is formed as a solid cellular structure (the so-called medullary cord). D and E : Dorso-ventral gradient of epithelialization. of development ${ }^{11}$. His plate 5 (Fig. 1) describing a chick embryo (probably at the 25-somite-stage since the tail fold is just formed) clearly shows what is known as secondary neurulation by cavitation. Gasser described there for the very first time to the best of my knowledge the presence of multiple lumens in the developing neural tube. He postulated that these different lumens merge to form the lumen of the caudal neural tube.

In 1882, Maximilian Braun (1850-1930) described the caudal region of sheep embryos in which the neural primordium is present as a solid cell structure (Fig. 2) located caudally to the neural tube ${ }^{2}$. He observed a few small cavities whose size is that of nuclei. Nowadays, we can wonder about these cavities: do they represent true intercellular spaces or are they generated by the dehydration of the sections linked to the fixation and then to the inclusion in paraffin?

Anyway, these two authors have shown the existence of a process of neurulation which is morphologically different from that of primary neurulation as early as the second part

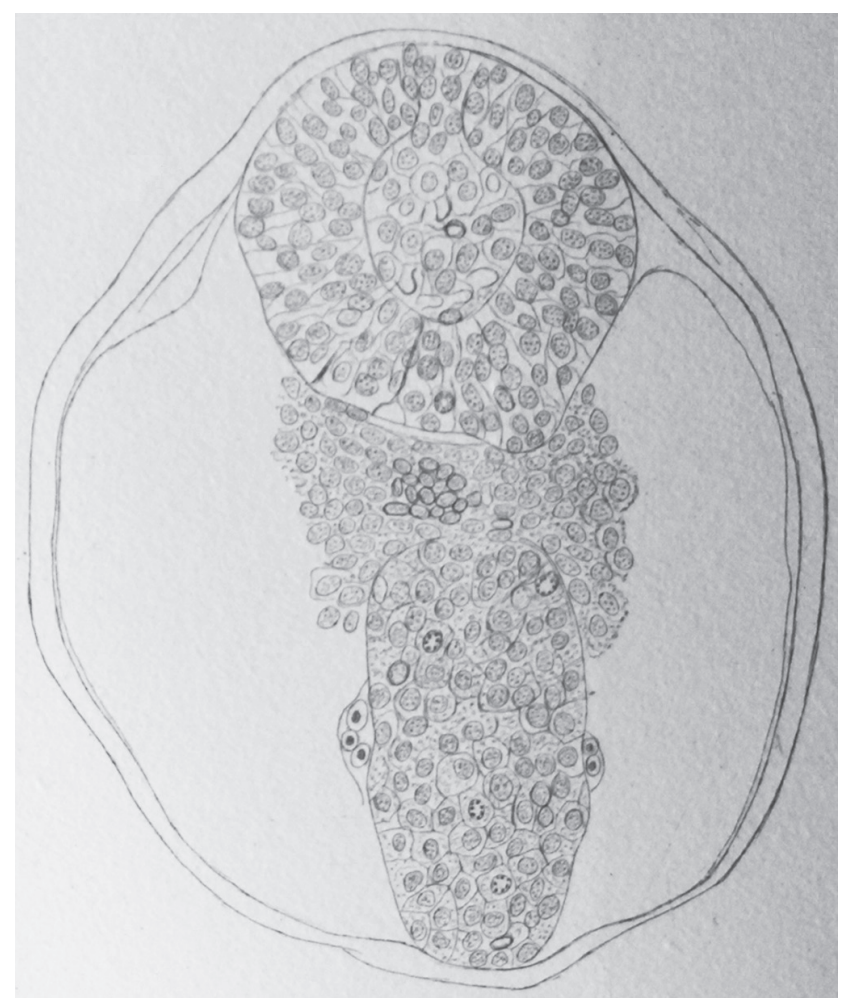

Fig. 2. Axial section of a sheep embryo (Maximilian Braun) ${ }^{4}$. The caudal neural tube (at the top of the figure) is formed by a solid rod of cells. Peripheral cells are epithelial whereas core cells are mesenchymal. This structure is now known as the medullary cord. 
of the 19th century.

\section{Secondary neurulation in chick embryos}

Undoubtedly, the species for which secondary neurulation is best described is chick ${ }^{7,8,16,32,35,37)}$. It is well known that the general development of the body of the vertebrate embryo follows a rostro-caudal sequence (or gradient). As a result, the rostral region is in advance of maturation compared to the caudal area. It is therefore generally accepted that analysing sections from an embryo in a caudo-rostral sequence provides information on the phenomena of maturation. This is why I will describe here the morphological aspect of the tail bud in chick, starting with the most caudal levels.

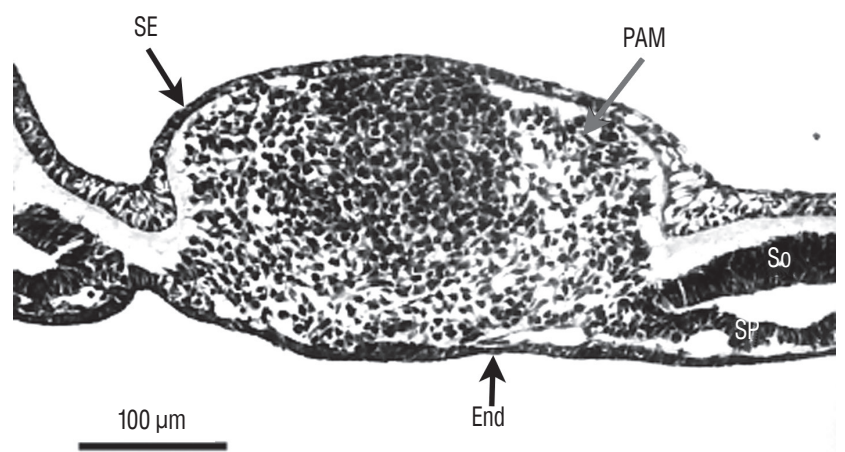

Fig. 3. Axial section of the caudal region of the tail bud (chick, 25-somite-stage). Tail bud cells are mesenchymal covered by surface ectoderm (SE). Their density is higher on midline. Laterally mesenchymal cells form the paraxial mesoderm (PAM). End : endoderm, So : somatopleural mesoderm, Sp : splanchnopleural mesoderm.

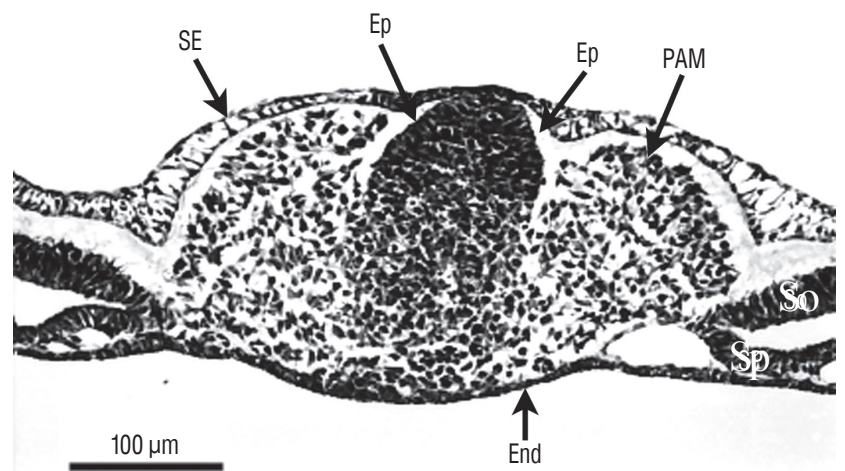

Fig. 4. Axial section involving a more rostral region of the tail bud than represented on Fig. 3 (chick, 25-somite-stage). Tail bud cells are sandwiched between surface ectoderm (SE) and endoderm (End). On the midline, dorsal cells aggregate to form an epithelium (Ep). In contrast, ventral cells are still mesenchymal. Paraxial mesoderm (PAM) is located laterally. So : somatopleural mesoderm, Sp : splanchnopleural mesoderm.
At the caudal level (Fig. 3), an axial section of the embryo shows mesenchymal tissue located between the surface ectoderm (forming the most dorsal region of the embryo) and the endoderm (its ventral region). There is no apparent differentiation, the only visible feature is the cell density which is greater in the midline than laterally. The lateral mesenchymal area represents the paraxial mesoderm.

More rostrally (Fig. 4), dorsal midline cells adopt an epithelial phenotype whereas ventral midline cells are still mesenchymal. This epithelial structure is surrounded by a basal lamina, ${ }^{9,40)}$ except for its ventral limit.

More rostrally (Fig. 5), neural midline cells appear as a ho-

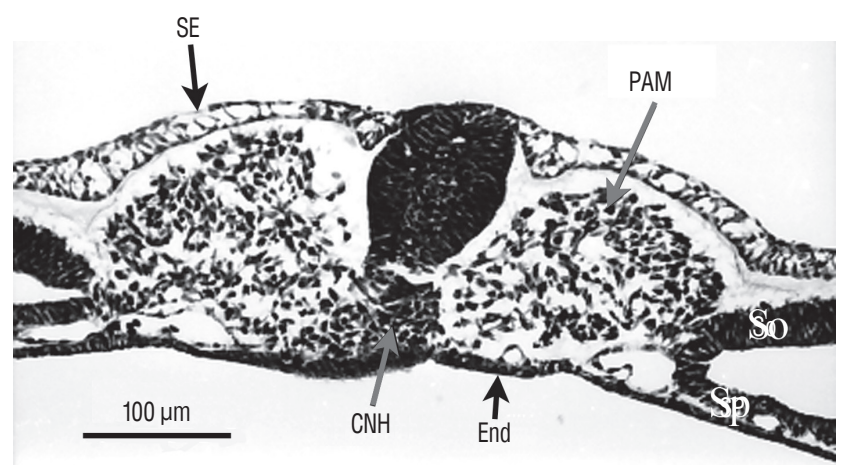

Fig. 5. Axial section of a more rostral region than that illustrated on Fig. 4. The tail bud is still limited by surface ectoderm (SE) dorsally and endoderm (End) ventrally. The histological aspect of midline cells has dramatically changes. Neural cells aggregate to form a solid rod of cells (the so-called medullary cord). Underneath this epithelial structure, lies the chordoneural hinge $(\mathrm{CNH})$ which is the region where notochord, ventral neural tube and mesenchyme of the tail bud merge. The space between $\mathrm{CNH}$ and ventral neural tissue is an artefact due to dehydration. Paraxial mesoderm (PAM) is located laterally. So : somatopleural mesoderm, Sp: splanchnopleural mesoderm.

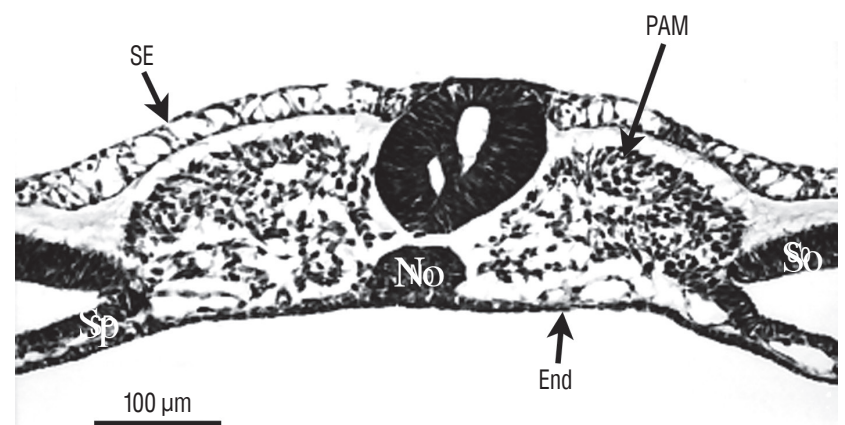

Fig. 6. Axial section from a more rostral level than represented on Fig. 5. The neural tube contains two lumens. The space between ventral neural tissue and notochord (No) is an artefact due to dehydration. SE : surface ectoderm, PAM : paraxial mesoderm, Sp : splanchnopleural mesoderm, End: endoderm, So: somatopleural mesoderm. 
mogeneous rod of cells (the so-called medullary cord). Underneath, an axial structure (or chordoneural hinge), which is fused with the neural tube and the tail bud mesenchyme, can be evidenced.

More rostrally (Fig. 6), two lumens develop inside the medullary cord. Underneath, notochord is adherent to the developing secondary neural tube.

More rostrally (Fig. 7), the lumens coalesce to generate the unique lumen of the secondary neural tube.

After fusion of the lumens (Fig. 8), the neural tube is morphologically similar to a neural tube produced by primary neurulation.

On sagittal section, notochord, neural tube and tail bud merge forming a region called the chordoneural hinge (Fig. 9).

In order to understand the relationships between all these different lumens and with the lumen of the more rostral neural tube, it is important to perform a $3 \mathrm{D}$ reconstruction of the caudal structure of the embryo. This was done by Schumach-

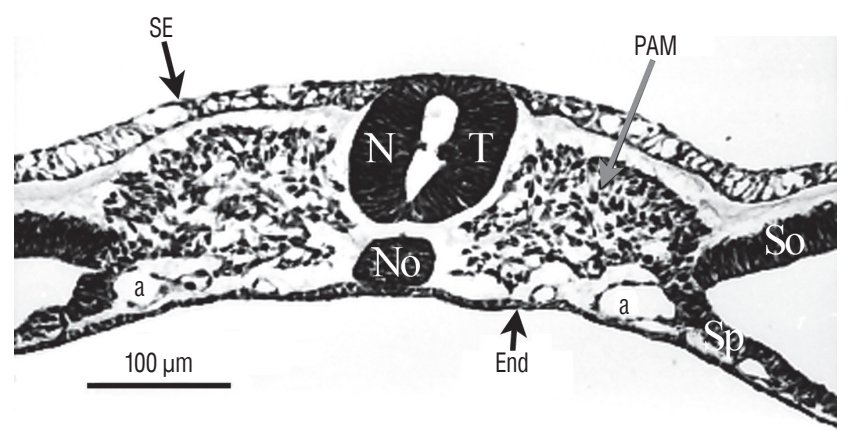

Fig. 7. At more rostral level compared to Fig. 6, the two lumens fuse. SE : surface ectoderm, NT : neural tube, PAM : paraxial mesoderm, a : aorta, No : notochord, End : endoderm, So : somatopleural mesoderm, Sp : splanchnopleural mesoderm.

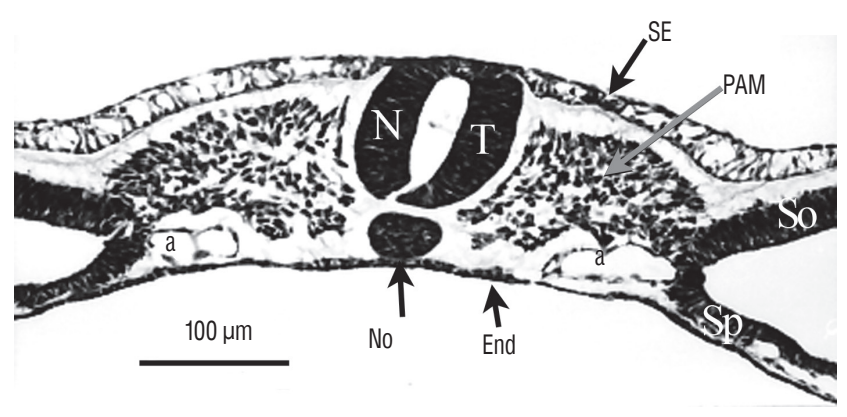

Fig. 8. At last, for more rostral levels, the neural tube (NT) formed by cavitation is strictly similar to a neural tube produced by primary neurulation. SE : surface ectoderm, PAM : paraxial mesoderm, a : aorta, No : notochord, End : endoderm, So : somatopleural mesoderm, Sp : splanchnopleural mesoderm. $\mathrm{er}^{37)}$ in chick embryo (Fig. 10). Following this reconstruction, the author demonstrates that all the secondary lumens are in relation with the lumen of the rostral neural tube from the onset of the formation of the caudal region. This result contrasts with the diagrams published in the reference textbooks. Indeed, in these works, the lumen is hollowed out within the tail bud initially unrelated to the more rostral lumen. According to these works, communication between the two lumens proceeds secondarily. So we see that such a pattern does not reflect reality at least with regard to chick.

The study of chick embryos younger than the stage that I

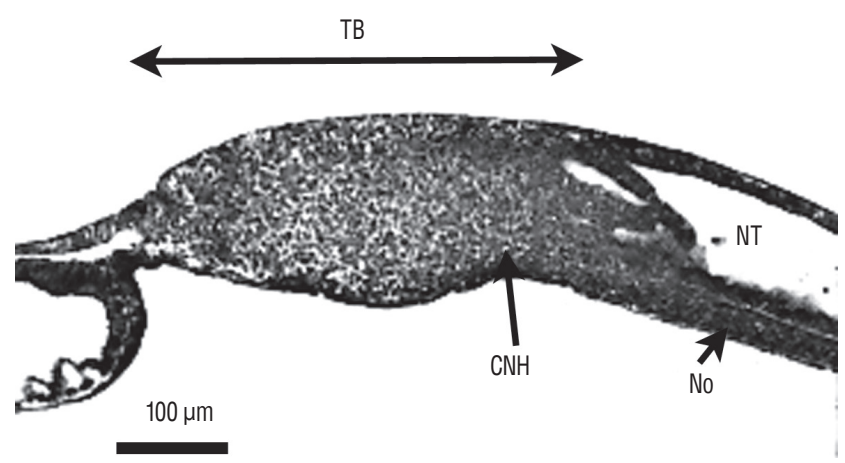

Fig. 9. Sagittal section of a chick embryo (24-somite-stage). The rostral pole is located at the right of the figure. The neural tube (NT) and underlying notochord (No) merge with tail bud (TB) mesenchyme forming the so-called chordoneural hinge (CNH).
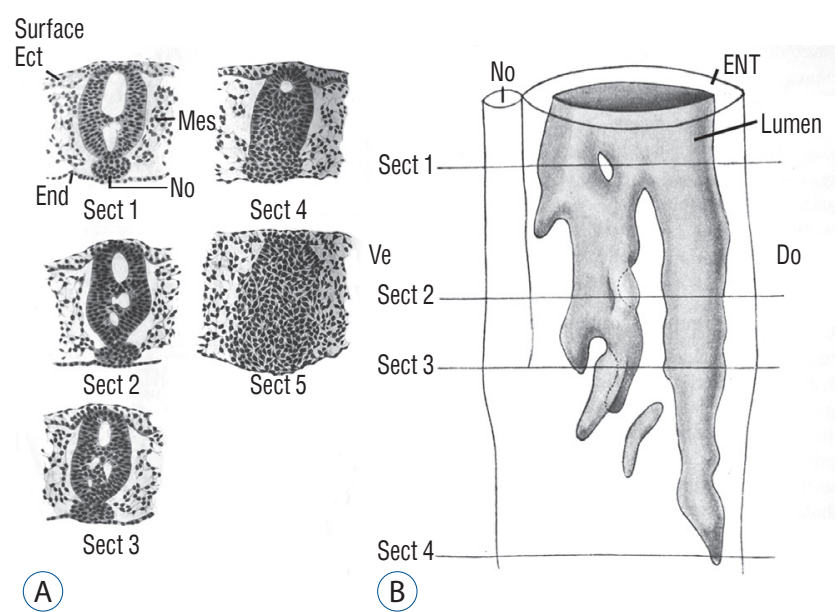

(B)

Fig. 10. A : Axial sections of a chick embryo showing progression of secondary neurulation. $B$ : These sections were used to perform 3D reconstruction. Following this reconstruction, we see that secondary lumens freely communicate with the primary lumen of the neural tube. Note that section 5 is located more caudally and consequently is not included on Fig. 10B. Modified from Schumacher ${ }^{37)}$. Ect : ectoderm, Mes: mesoderm, End : endoderm, No : notochord, ENT : epithelium of the neural tube, Do : dorsal, Ve : ventral. 
have just described, shows an overlap zone where the neural tube is formed by primary neurulation in its dorsal part while its ventral part is formed by secondary neurulation. This singular aspect has been called junctional neurulation ${ }^{8,9)}$. I will not describe further this process; this is another story.

\section{Secondary neurulation in mouse embryos}

Surprisingly enough, few studies have been devoted to secondary neurulation in mouse. The goal and the methods used in these studies were diverse : histological descriptions ${ }^{33,36)}$, mode of development of the basal lamina ${ }^{26}$, and distribution of cell surface glycoconjugates ${ }^{14)}$. No overlap zone between primary and secondary neurulation is observed in mouse contrarily to chick ${ }^{33,36)}$. The lumens of the primary and secondary neural tubes are continuous from the onset of secondary neurulation ${ }^{25,26,33,36)}$. This feature is particularly well analysed on sagittal sections. Multiple lumens are never observed in this species contrarily to what is commonly observed in chick embryo ${ }^{14,33,36)}$. As in chick embryo, dorsal cells first epithelialize with gradual incorporation of more ventral cells ${ }^{26,33}$, this chronology explains why basal lamina is first dorsally situated $^{26)}$.

\section{Secondary neurulation in human embryos}

Several studies have focused on the caudal region of the human embryo. The age of embryos was only defined by crownrump length (CRL) in older studies ${ }^{21,37}$. For the others, the age was established according to the criteria of the Carnegie classification $^{22-25,29,45)}$. The age of the embryos used by the first two authors can be imperfectly estimated, although this estimate should be taken with caution. The posterior neuropore closes at Carnegie stage $12^{23,25,29)}$. At this moment of development, Saitsu et al. ${ }^{29)}$ illustrate and describe a condensed axial mesenchymal mass located between the primary neural tube (dorsal) and the notochord (ventral). This histological aspect recalls that of the zone of overlap between primary and secondary neurulations described in chick. Müller and O'Rahilly ${ }^{23)}$ claim that no area of overlap can be observed. The $4 \mathrm{~mm}$-long embryo illustrated by Kunitomo ${ }^{21)}$ appears to have an area of overlap even though the author does not describe it. At stage 13 , the appearance of overlap between the two types of neurulations is no longer present ${ }^{29)}$. The lumens produced during primary and secondary neurulations are continuous ${ }^{22-25,29,37)}$ (Fig. 11). It is important to note that cavitation does not occur independently in the tail bud followed by a secondary aperture of the lumen in the primary neural tube as very often illustrated in the reference textbooks. At the onset of secondary neurulation, there are never multiple lumens visible in the human caudal neural tube. For some authors ${ }^{24)}$, multi-cavitation is never observed. For others, multiple cavities are present from stage $13^{29)}$ or stage $14^{22,45)}$.

\section{Secondary neurulation in other mammals}

Fragmentary data have been published for $\mathrm{rat}^{20)}, \mathrm{pig}^{17)}$, and Golden Syrian hamster ${ }^{38}$. The sole available data we gained by reading these references is that a unique lumen develops for these species.

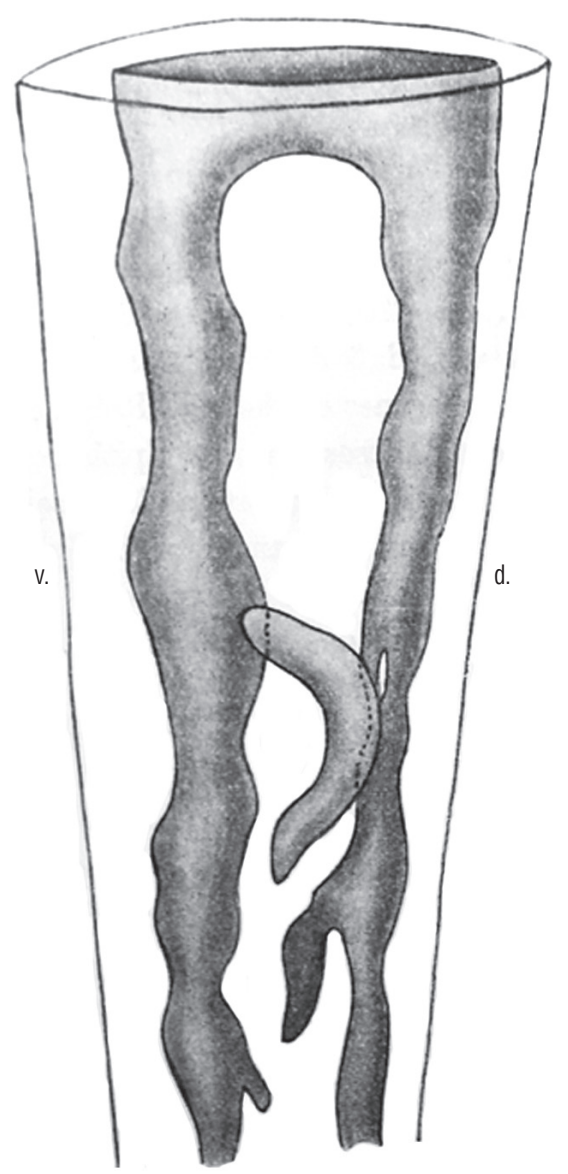

Fig. 11. 3D reconstruction of a caudal neural tube from a human embryo ( $8 \mathrm{~mm}$-long, probably Carnegie stage 15 . The multiple lumens (grey) are communicating with the rostal neural tube. Modified from Schumacher ${ }^{37)}$ d : dorsal, v : ventral. 


\section{TWO HYPOTEHSES CONCERNING THE MODE OF GROWTH AND DIFFERENTIATION OF THE TAIL BUD}

David Holmdahl (1887-1956) describes the chick embryo at different ages ${ }^{16}$. From the histological appearance of the tail bud, he postulates that the embryonic body is formed by two radically different ways. The primary formation of the body involves transient structures that are the primordial layers (ectoderm, mesoderm and endoderm). From the neural ectoderm, the primary neural tube forms and gives rise to the rostral end of the central nervous system. The tail bud, on the other hand, does not develop features revealing primordial layers and Holmdahl proposes a direct derivation of the caudal embryonic structures without passing through the primordial layers. He considers the tail bud as a "blastema" made up of homogeneous and undifferentiated cells. This mode of body formation is called secondary formation and it is materialized by the phenomenon of secondary neurulation.

Jean Pasteels (1906-1991) follows a more comparative approach and describes the caudal end of many vertebrate species. For birds, no histological differentiation should be evident $^{27)}$. Nevertheless, he compares the observed appearance to that of amphibians for which experimental data are available at that time. Since the works of Walter Vogt (1888-1941), it has indeed been possible to use vital dyes to stain and follow the fate of cells in these species. This mode of experimentation shows that the caudal bud in amphibians is formed by a mosaic of juxtaposed territories ${ }^{2}$. Pasteels postulates that it is the same in birds and that the histological undifferentiation reflects only appearances.

\section{HETEROGENEITY OF THE TAIL BUD}

On the one hand, the tail bud of birds and mammals has long been considered a blastema ${ }^{15)}$ according to the model proposed by Holmdahl. On the other hand, the caudal region of amphibians was seen as a mosaic of territories. Such a conception of the mammalian tail bud is still widely used in the medical literature or in reference textbooks of human developmental biology. This blastema theory explains why the first experimental studies concerning the tail bud of birds always considered this bud as a homogeneous structure. Microsurgi- cal removal of the entire bud results in a developmental failure of the embryonic tail in birds ${ }^{31,46)}$. It is interesting to note that the caudal notochord is still normal after these ablations ${ }^{31}$. Nevertheless, after such surgical removal, the tail can form normally showing the regenerative capacities of this region $^{31,46)}$. This regeneration phenomenon makes interpretations of these experimental studies difficult. Therefore, further experimental studies were needed to study the fate of tail bud in birds. They involve homotopic and isochronic transplants (the graft replaces the same anatomical region of the host, host and donor have the same age) that were either marked with tritiated thymidine ${ }^{30)}$ or by using the chimeric quail chicken system $^{34)}$, a system that I will describe later. The graft gives rise to the caudal neural tube, the caudal somites but never to the notochord and to the endodermal structures. However, as the tail bud is transplanted en bloc, it is not possible to demonstrate heterogeneity in the grafted structure using this type of experiments.

\section{A fate mapping experiment in birds}

In order to determine if the tail bud of birds is a homogeneous structure as imagined by David Holmdahl or if, on the contrary, it is composed by different territories (according to Jean Pasteels), we realized a fate map of this region in birds ${ }^{7}$. To do this, we used the quail-chick chimera model developed by Nicole Le Douarin. Quail cells can be identified from chick cells. Two techniques make it possible to differentiate them : Feulgen-Rossenbeck reaction stains their heterochromatin, which is dense and associated with the nucleolus in quails, while it is more diffuse in chicks. More recently, an antibody recognizes the nucleus of quail cells but not the chick one. Consequently, if you transplant a region taken from a quail embryo and replace the homologous region in the chick embryo, you can create an interspecific chimera that develops. At the end of this development, one can determine the contribution of the graft to the chimeric organism and therefore unveil the fate of a specific region of the embryo.

In our work, we subdivided the tail bud of an embryo with 25 pairs of somites into four sub-regions ${ }^{7}$. I will only deal here with the three regions of the medial part of the embryo because the fourth (more lateral) region is involved in the genesis of vertebral structures and does not participate in the formation of the secondary neural tube. At this stage, the neural tube is closed, recognizable by its lumen. The tail bud lies di- 
rectly in continuity with the neural tube. The first region (Fig. 12) we study corresponds to the most caudal part of the already formed neural tube. Ventrally, this region is in relation with the notochord rostrally and with the chordoneural hinge caudally. The tissues located caudally to this region are separated into two sub-regions. The rostral two thirds constitute the second region of interest, the third is represented by the caudal third (Fig. 12).

If we graft the neural tube of the first region without grafting either the notochord or the chordoneural hinge, we observe that the transplanted tissue has generated the spinal cord corresponding to the level of the sacral nerves 3 to 6 as well as to the structures derived from the neural crest originating from these levels. If we transplant the neural tube and the dorsal part of the chordoneural hinge, we note that, for the spinal cord located caudally to the 6th sacral nerves, the graft generated the floor plate of the spinal cord, the rest of this region coming from the host. Finally, if the graft contains the neural tube and all the chordoneural hinge, the caudal notochord (from sacral region to the tip of the tail) also derives from the donor adding to the previous contribution (Fig. 13). Thus, we have shown that the chordoneural hinge participates in the formation of the caudal notochord and the secondary neural tube and that it is the exclusive origin of the floor plate of the neural tube. It therefore represents the equivalent of Hensen's node in more rostral regions.

The second region of interest gives rise to nervous but also

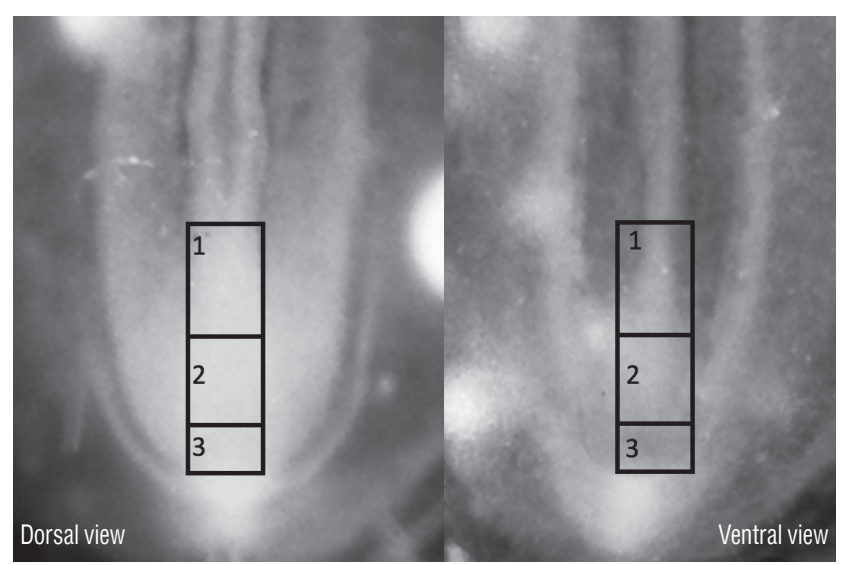

Fig. 12. The three regions that have been transplanted in the avian tail bud. Region 1 corresponds to the most caudal part of the neural tube and notochord. Furthermore, the chordoneural hinge is also involved in this experiment. Region 2 corresponds to $2 / 3$ rostral of the tail bud whereas region 3 to its caudal $1 / 3$. vertebral derivatives. Transplantation of this region yields the spinal cord of the caudal regions from the level of the 7 th pair of sacral nerves. The portion of spinal cord produced by this region is complementary to that generated by the more rostral region: the entire spinal cord derives from the graft excluding the floor plate of the neural tube (Fig. 14). In addition, transplanted cells participate in the formation of vertebral structures (i.e., caudal synsacrum, free caudal vertebrae and pygostyle in chick) which constitute the caudal end of the spine in birds.

Finally, the third region that we have delimited contributes to the formation of the caudal vertebral structures in collaboration with the immediately rostral region. It is important to note that this most caudal region of the tail bud does not generate any cells forming the spinal cord.

In conclusion, the major result obtained in this study is the demonstration of heterogeneity within the tail bud in the avian embryo. These data rather argue in favour of Jean Pasteels'

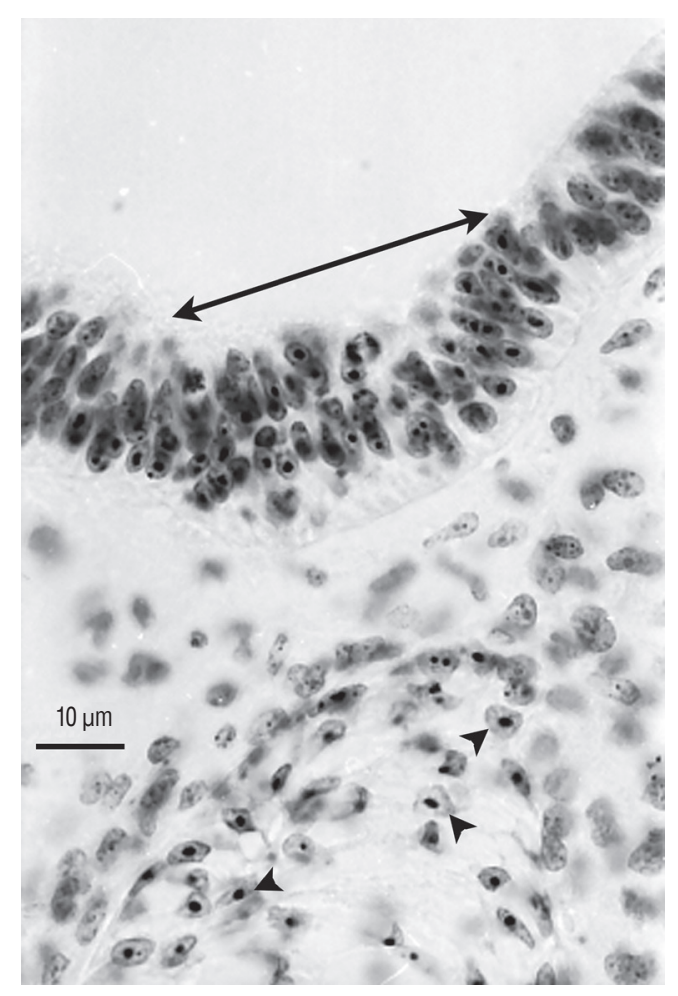

Fig. 13. Axial section of a chimera for which a quail region 1 was transplanted into a chick host. Quail cells can be recognised by their condensed heterochromatin (arrowheads). Caudally to the level of sacral nerves 3 to 6 , the graft yields the caudal notochord (arrowheads) and the floor plate (double arrow). All the rest of the neural tube is derived from the host (Feulgen-Rossenbeck staining). 
model and allow to rule out the model of a homogeneous blastema made of pluripotent cells as proposed by David Holmdahl.

Such a heterogeneity of the tail bud could be revealed by gene expression pattern. Indeed, some genes are not widely expressed by all the tail bud but by a limited number of cells suggesting heterogeneity at the genetic level. Such is the case in zebrafish ${ }^{3)}$, Xenopus ${ }^{1,13)}$, chick $^{19)}$, and mouse ${ }^{12,44)}$. Fate map

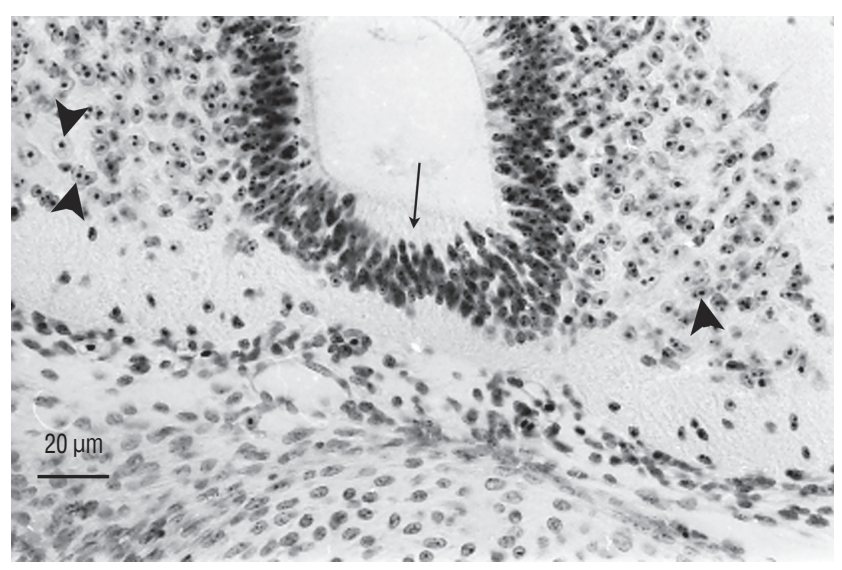

Fig. 14. Axial section of a chimera for which a quail region 2 was transplanted into a chick host. The spinal cord, located caudally to the level of sacral nerves 6 , derived from the graft (arrowheads pointing to cells with a condensed heterochromatin) except for its floor plate (arrow) that is produced by the host (Feulgen-Rossenbeck staining). Comparing Figs. 13 and 14 shows the complementary pattern observed after grafting.

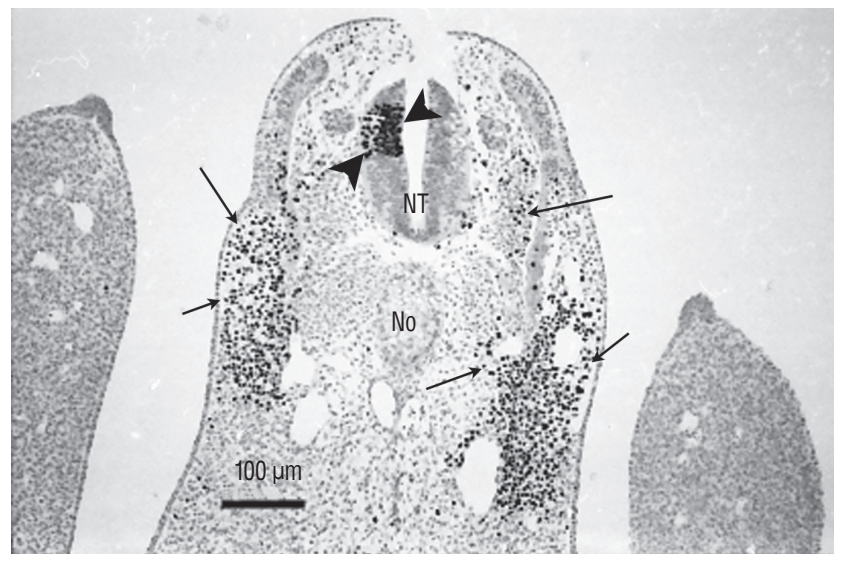

Fig. 15. Section of a chimera in which a quail region 2 was placed at the level of region 3 in a chick host. QCPN antibody marks specifically the quail nucleus and not the chick one. Grafted cells contribute to neural tube (NT) (arrowheads) and somitic derivatives (arrows). This heterotopic transplantation shows that the fate of the grafted region is not modified by its new environment (Immunohistochemistry using QCPN antibody). No: notochord. studies in other vertebrates also show that the tail bud is in fact composed by a mosaic of territories whose fate is different: such is the case for zebrafish ${ }^{10,18,28,39)}$, Xenopus ${ }^{13,42)}$, and mouse ${ }^{5,43}$. Such conservation in members of the principal phyla of vertebrates highly suggests that this is a conserved feature during phylogenesis. This strongly suggests that such a heterogeneity in the tail bud should also apply to humans.

\section{Heterotopic transplantations}

However, this heterogeneity might only reflect diversity of inductions. For example, we noted that the most caudal region of the tail bud only gives rise to mesodermal derivatives. This restriction of developmental potentials could be explained by a limited capacity for differentiation or by a lack of neural induction. To test this phenomenon, I performed heterotopic transplants (in which the grafted region is placed in a different environment). Thus, if the most caudal region of a quail embryo (region 3) is transplanted into the intermediate region of a chick embryo (region 2), grafted cells will be subjected to conditions which allow the emergence of a neural phenotype in the host. If the graft transplanted in a heterotopic position can generate neural cells, then we will have evidence that the differentiation potentials of the caudal region is restricted by lack of induction. On the contrary, if the transplanted cells cannot produce neural cells but only mesodermal cells, then we will have a strong indication on the mesodermal determi-

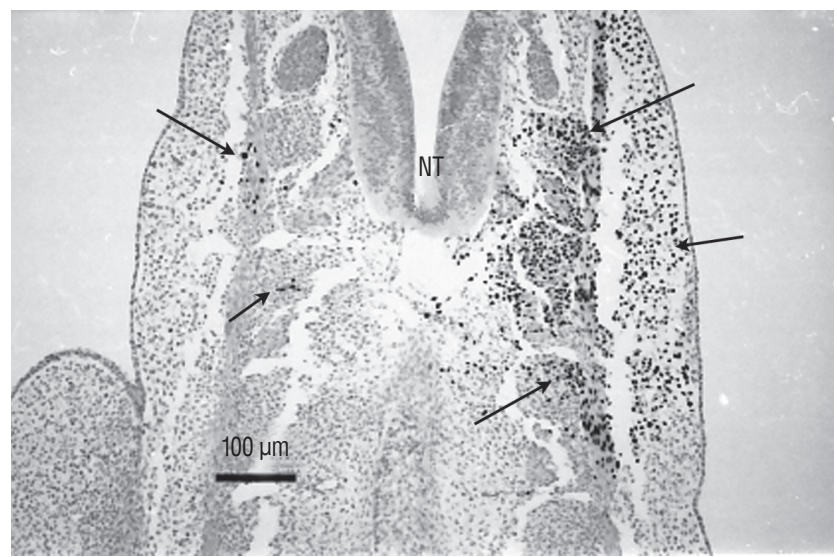

Fig. 16. Section of a chimera in which a quail region 3 was placed at the level of region 2 in a chick host. QCPN antibody marks specifically the quail nucleus and not the chick one. Grafted cells contribute to somitic derivatives (arrows). There is no contribution of grafted cells to the neural tube (NT). This heterotopic transplantation shows that the fate of the grafted region is not modified by its new environment (Immunohistochemistry using QCPN antibody). 
nation of this region. The results show that the intermediate region of the tail bud (which displays both neural and mesodermal potentialities) transplanted into the most caudal region generates derivatives in neural tube and somites (Fig. 15). Likewise, the most caudal region (which develops only mesodermal derivatives) transplanted more rostrally does not generate neural derivatives but only somitic ones (Fig. 16). Thus, the restriction of developmental potentials of the caudal region is determined and cannot be changed even if the latter is placed in a permissive environment. Regarding the intermediate region, two possibilities remain : either bipotential cells (both neural and mesodermal) are present or this region is made up by a mosaic of territories. Similar results have been described for the mouse embryo ${ }^{5)}$. In this journal, Yoshiko Takahashi (Kyoto, Japan) presents a more specific review on the subject of cell fate in this region.

\section{SUPERFICAL (ECTODERMAL) ORIGIN OF CAU- DAL SPINAL CORD CELLS}

It is generally widely accepted that secondary neurulation abnormalities generate by definition closed neural tube defects. Consequently, an open neural tube defect suggests that it is due to a process affecting primary neurulation. If this assumption is true, reported cases of craniorachischisis largely involving the sacral region would suggest that the spinal cord of these regions should be formed by primary neurulation. However, this conception seems excessive to us according to our already published results.

In order to determine the relationships between primary and secondary neurulation in birds, we carried out a fate map of the territories of the rhomboid sinus ${ }^{6)}$. At the 6 somite stage in birds (Fig. 17), the neural plate is still open. At the caudal end of the notochord and in the centre of the neural plate, a depression is clearly visible, representing Hensen's node as it stands during its regression. Using the quail-chicken chimera technique, we have demonstrated that Hensen's node gives rise to the notochord as well as the floor plate of the neural tube down to the caudal end of the embryonic tail. Thus we demonstrate that Hensen's node generates the chordoneural hinge and that the process of axial extension of the ventral midline is continuous between the two types of neurulation. Furthermore, if the latero-caudal region of the neural plate of

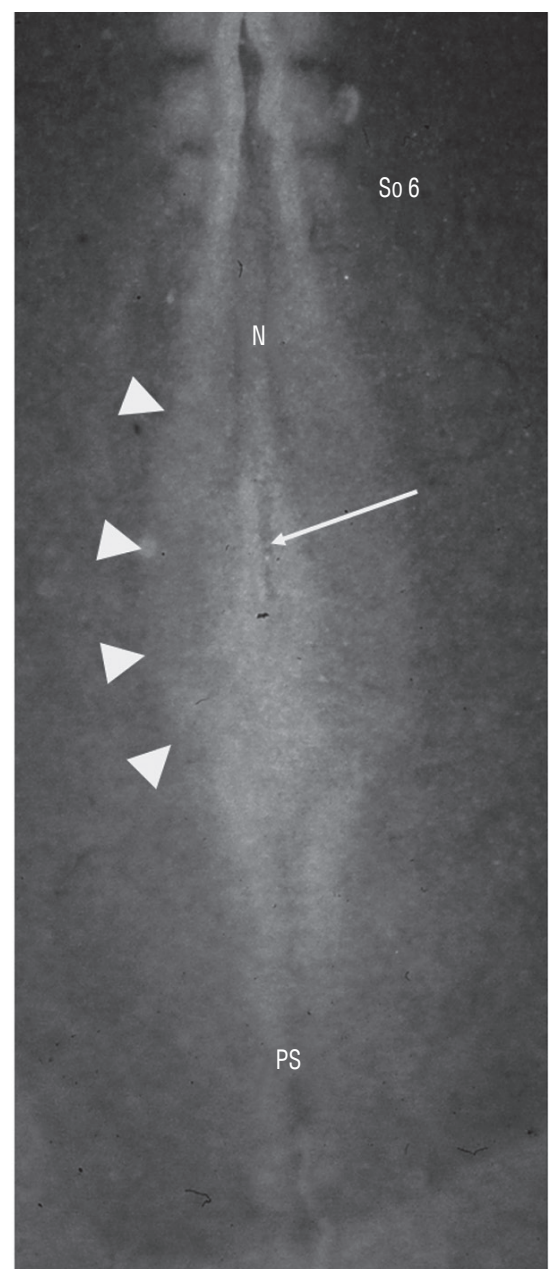

Fig. 17. Dorsal view of a chick embryo at 6 somite (So)-stage. Neurectoderm is still largely open forming a neural plate (arrowheads). At the caudal end of notochord $(\mathrm{N})$, lies a depression corresponding to regressing Hensen's node (arrow). PS : primitive streak.

a quail is transplanted into a donor chick, the graft generates part of the secondary neural tube (Fig. 18). These results, suggesting that the tissues (or at least part of them) generating the caudal spinal cord by secondary neurulation are located in the superficial layer of the embryo, have been largely ignored by the medical literature.

More recently, we have studied the process of junctional neurulation". The caudal neural plate was labelled with the carboxyfluorescein diacetate succimidyl ester (CFDA-SE). This dye penetrates the superficial cells of the embryo. In cytoplasm, the dye is transformed by cellular enzymes into carobyfluorescein succinmidyl ester (CFSE) which remains in the cytoplasm and cannot leave the cell. It is therefore a way to mark the most superficial cells and to follow their fate. After 

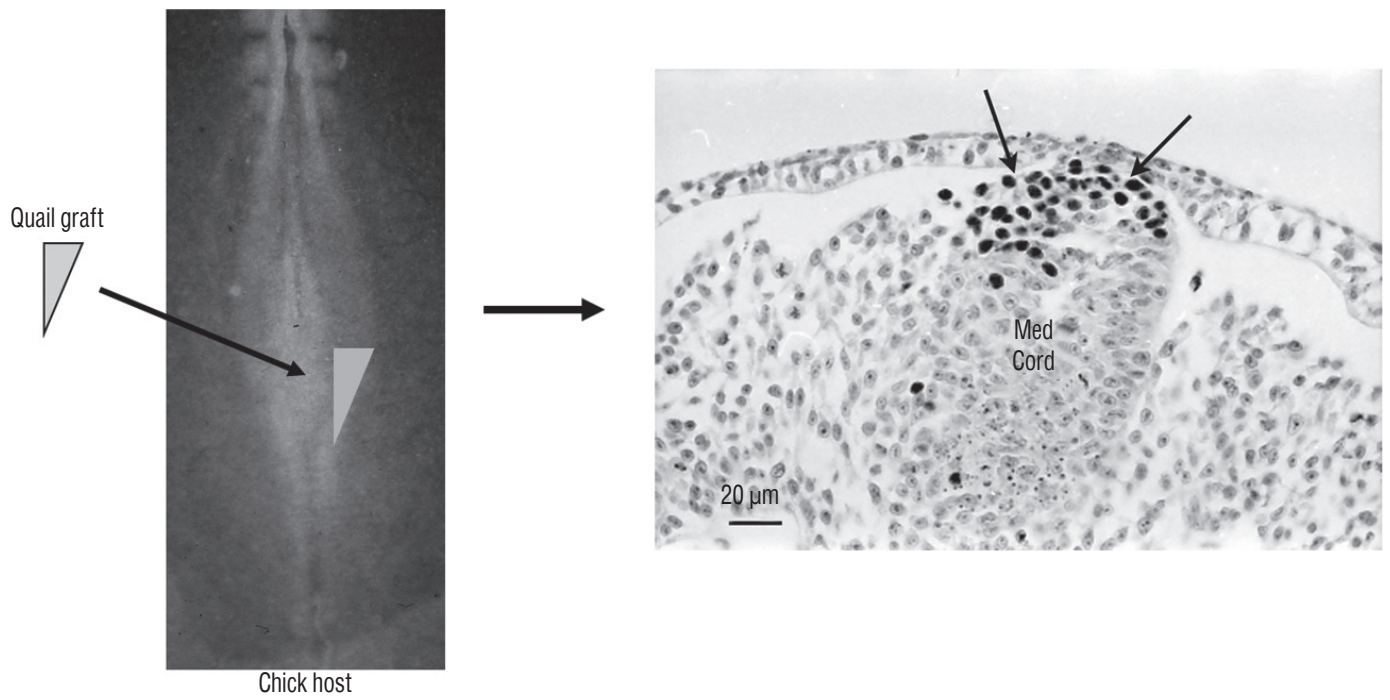

Fig. 18. A graft containing the latero-caudal part of the neural plate from a 6-somite quail embryo is placed at the same level in a chick host. After inculation, grafted cells (recognized by QCPN antibody) develop and give riose to the dorsal part of the medullary cord (Med Cord) (arrows). Modified from Catala et al. ${ }^{6}$.

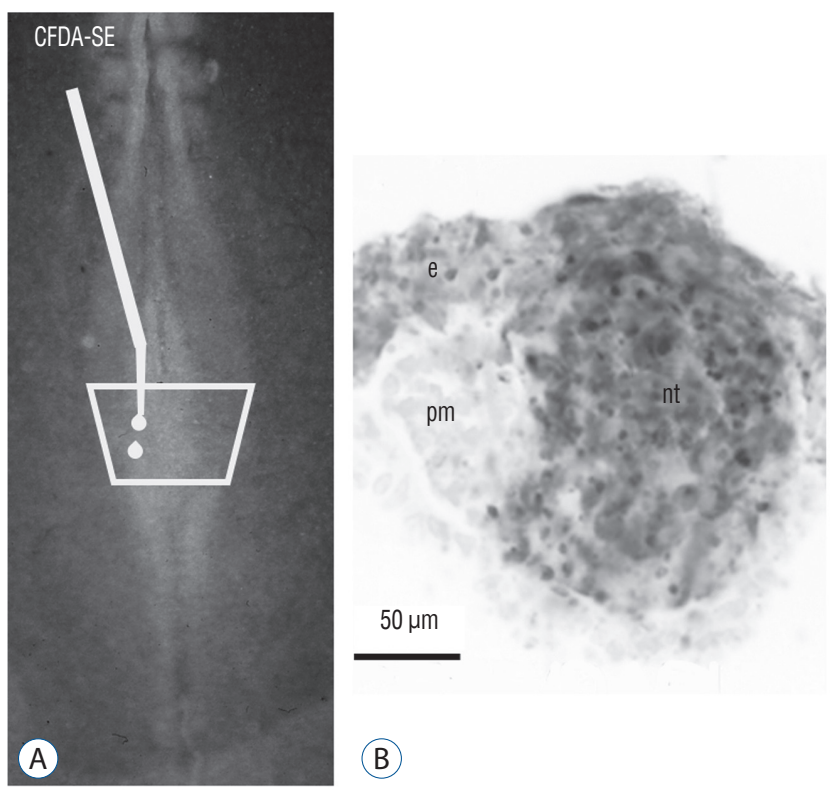

Fig. 19. A and $B$ : The superficial neural plate of a 6 somite chick embryo is stained by CFDA-SE. After incubation, marked cells (in grey) generate superficial ectoderm (e) and the secondary neural tube (nt). The paraxial mesoderm (pm) is not stained. Modified from Dady et al. ${ }^{9}$.

staining the caudal neural plate of a 6 somite chick embryo, the entire medullary cord (precursor of the secondary neural tube) is stained (Fig. 19). This second experiment validates our first result and proves that all the tissues that would generate the spinal cord by secondary neurulation are located in the superficial layer of the embryo. In addition, we have shown that the internalization of these cells occurs by ingression, that is, by epithelial-mesenchymal transition. Such cell movement was previously described only for the mesoderm and endoderm during gastrulation. We thus show that part of the central nervous system also undergoes this same process. The final formation of the secondary neurectoderm supposes a second mesenchymal-epithelial transition. These results indicate that an abnormal ingression of the superficial neural tissues can lead to an open malformation even if the involved region is produced by secondary neurulation.

Such a superficial origin of tissues fated to formed caudal spinal cord has also been demonstrated for zebrafish ${ }^{18)}$ and amphibans ${ }^{2,41,42)}$. Such a mechanism has not been demonstrated in mammals and should lead my readers to remain cautious about the generalization of this phenomenon to all vertebrate species. Nevertheless, I believe that it would be very useful to design this type of experiments for mammalian embryos. This is, in my opinion, the sole way to demonstrate that this process is conserved during phylogenesis.

\section{CONCLUSIONS}

The formation of the neural tube (also known as neurulation), the precursor of the central nervous system, is an im- 
portant stage in the development of the embryo. In most of the textbooks, this step is presented in a simplistic way without taking into account the very complex dimension of this process and the diversity of the mechanisms developed along the rostro-caudal axis. The caudal part of the spinal cord forms after the posterior neuropore closes by a morphologically different mechanism called secondary neurulation. This process is well studied in birds but its knowledge is less complete concerning mammals. In humans, purely descriptive data are available. However, they are contradictory, calling for the greatest caution before using them to unveil the mechanisms of caudal malformations. Only a developmental biologic approach centered on caudal morphogenetic processes could provide a better understanding of morphological aspects in humans. However, analysis of the results obtained in many vertebrate species shows that the model commonly used to understand caudal malformations in humans is inaccurate. The tail bud is not a pluripotent structure made up of undifferentiated cells but should be considered as a mosaic of territories. The lumens of the primary and secondary tubes are continuous during their formation and there is no secondary opening of the caudal lumen into the more rostral region. Initially during development, tissues that are fated to form neural tube by secondary neurulation are located superficially. They need to internalize for undergoing neurulation; if this process is impaired, a defect affecting secondary neurulation would produce open neural tube defect. All these data gained in developmental biology argue for proceeding with a "re-reading" of human malformations; this re-reading should, in my opinion, only be carried out within multidisciplinary teams (neurosurgeons, radiologists, pathologists, geneticists, and of course developmental biologists).

\section{CONFLICTS OF INTEREST}

No potential conflict of interest relevant to this article was reported.

\section{INFORMED CONSENT}

This type of study does not require informed consent.

\section{AUTHOR CONTRIBUTIONS}

\author{
Conceptualization : MC \\ Data curation : MC \\ Formal analysis : $\mathrm{MC}$ \\ Funding acquisition : $\mathrm{MC}$ \\ Methodology : MC \\ Project administration : MC \\ Visualization: MC \\ Writing - original draft : MC \\ Writing - review \& editing : MC
}

\section{ORCID}

Martin Catala https://orcid.org/0000-0003-1554-9007

\section{- Acknowledgements}

I thank all the people with whom I have worked on neurulation during the last 33 years : Nicole Le Douarin, Marie-Aimée Teillet, Eddy De Robertis, Jean-Loup Duband and Alwyn Dady. I gratefully thank the "Bibliothèque Inter-Universitaire de l'Universite de Paris" and all the people working in this fantastic place for giving me the opportunity to read historical papers on neurulation and to allow me reproducing the historical illustrations of this paper.

\section{References}

1. Beck CW, Slack JM : Analysis of the developing Xenopus tail bud reveals separate phases of gene expression during determination and outgrowth. Mech Dev 72 : 41-52, 1998

2. Bijtel JH : Über die Entwicklung des Schwanzes bei Amphibien. Wilhelm Roux Arch Entwickl Mech Org 125 : 448-486, 1931

3. Bouldin CM, Manning AJ, Peng YH, Farr GH 3rd, Hung KL, Dong A, et al. : Wnt signaling and tbx16 form a bistable switch to commit bipotential progenitors to mesoderm. Development 142 : 2499-2507, 2015

4. Braun $M$ : Entwickelungsvorgänge am Schwanzende bei einigen Säugethieren mit Berücksichtigungder Verhältnisse beim Menschen. Arch f Anat u Phys, Anat Abt 6 : 207-241, 1882

5. Cambray N, Wilson $V$ : Axial progenitors with extensive potency are localised to the mouse chordoneural hinge. Development 129 : 48554866, 2002

6. Catala M, Teillet MA, De Robertis EM, Le Douarin ML : A spinal cord fate map in the avian embryo: while regressing, Hensen's node lays down 
the notochord and floor plate thus joining the spinal cord lateral walls. Development 122 : 2599-2610, 1996

7. Catala M, Teillet MA, Le Douarin NM : Organization and development of the tail bud analyzed with the quail-chick chimaera system. Mech Dev $51: 51-65,1995$

8. Criley BB : Analysis of the embryonic sources and mechanisms of development of posterior levels of chick neural tubes. J Morphol 128 : 465501, 1969

9. Dady A, Havis E, Escriou V, Catala M, Duband JL : Junctional neurulation: a unique developmental program shaping a discrete region of the spinal cord highly susceptible to neural tube defects. J Neurosci 34 : 13208-13221, 2014

10. Davis RL, Kirschner MW : The fate of cells in the tailbud of Xenopus laevis. Development 127 : 255-267, 2000

11. Gasser E : Der Primitivstreifen bei Vogelembryonen (Huhn und Gans). Schriften der Gesellschaft zur Beförderung der gesammten Naturwissenschaften zu Marburg 2 (Suppl 1) : 1-98, 1879

12. Gofflot F, Hall M, Morriss-Kay GM : Genetic patterning of the developing mouse tail at the time of posterior neuropore closure. Dev Dyn 210 : 431-445, 1997

13. Gont LK, Steinbeisser H, Blumberg B, de Robertis EM : Tail formation as a continuation of gastrulation: the multiple cell populations of the Xenopus tailbud derive from the late blastopore lip. Development 119 : 991-1004, 1993

14. Griffith CM, Wiley MJ : The distribution of cell surface glycoconjugates during mouse secondary neurulation. Anat Embryol (Berl) 180 : 567575, 1989

15. Griffith CM, Wiley MJ, Sanders EJ : The vertebrate tail bud: three germ layers from one tissue. Anat Embryol (Berl) 185 : 101-113, 1992

16. Holmdahl DE : Die zweifache Bildungsweise des zentralen Nervensystem bei den Wirbeltieren. Eine formgeschichtliche und materialgeschichtliche Analyse. Wilhelm Roux Arch Entwickl Mech Org 129 : 206-254, 1933

17. Hughes AF, Freeman RB : Comparative remarks on the development of the tail cord among higher vertebrates. J Embryol Exp Morphol 32 : 355-363, 1974

18. Kanki JP, Ho RK : The development of the posterior body in zebrafish. Development $124: 881-893,1997$

19. Knezevic $V$, De Santo R, Mackem $S$ : Continuing organizer function during chick tail development. Development 125 : 1791-1801, 1998

20. Kostović-Knezević L, Gajović S, Svajger A : Morphogenetic features in the tail region of the rat embryo. Int J Dev Biol 35 : 191-195, 1991

21. Kunitomo $\mathrm{K}$ : The development and reduction of the tail and of the caudal end of the spinal cord. Contrib Embryol 8 : 161-198, 1918

22. Lemire RJ : Variations in development of the caudal neural tube in human embryos (horizons XIV-XXI). Teratology 2 : 361-369, 1969

23. Müller $F, O^{\prime}$ Rahilly $R$ : The development of the human brain, the closure of the caudal neuropore, and the beginning of secondary neurulation at stage 12. Anat Embryol (Berl) $176:$ 413-430, 1987

24. Müller F, O'Rahilly $R$ : The development of the human brain from a closed neural tube at stage 13. Anat Embryol (Berl) 177 : 203-224,
1988

25. Nievelstein RA, Hartwig NG, Vermeij-Keers C, Valk J : Embryonic development of the mammalian caudal neural tube. Teratology $48: 21-31$, 1993

26. O'Shea KS : Differential deposition of basement membrane components during formation of the caudal neural tube in the mouse embryo. Development 99 : 509-519, 1987

27. Pasteels J : Etudes sur la gastrulation des vertébrés méroblastiques. III. Oiseaux. IV Conclusions générales. Arch Biol 48 : 381-488, 1937

28. Row RH, Tsotras SR, Goto H, Martin BL : The zebrafish tailbud contains two independent populations of midline progenitor cells that maintain long-term germ layer plasticity and differentiate in response to local signaling cues. Development 143 : 244-254, 2016

29. Saitsu H, Yamada S, Uwabe C, Ishibashi M, Shiota K : Development of the posterior neural tube in human embryos. Anat Embryol (Berl) 209 : 107-117, 2004

30. Schoenwolf GC : Tail (end) bud contributions to the posterior region of the chick embryo. J Exp Zool 201 : 227-245, 1977

31. Schoenwolf GC : Effects of complete tail bud extirpation on early development of the posterior region of the chick embryo. Anat Rec 192 : 289-295, 1978

32. Schoenwolf GC : Histological and ultrastructural observations of tail bud formation in the chick embryo. Anat Rec 193 : 131-147, 1979

33. Schoenwolf GC: Histological and ultrastructural studies of secondary neurulation in mouse embryos. Am J Anat 169 : 361-376, 1984

34. Schoenwolf GC, Chandler NB, Smith JL: Analysis of the origins and early fates of neural crest cells in caudal regions of avian embryos. Dev Biol $110: 467-479,1985$

35. Schoenwolf GC, Delongo J : Ultrastructure of secondary neurulation in the chick embryo. Am J Anat $158:$ 43-63, 1980

36. Schoenwolf GC, Nichols DH : Histological and ultrastructural studies on the origin of caudal neural crest cells in mouse embryos. J Comp Neurol 222 : 496-505, 1984

37. Schumacher $S$ : Über die sogenannte Vervielfachung des Medullarrohres (bzw. des Canalis centralis) bei Embryonen. Z Mikrosk Anat Forsch $10: 83-109,1927$

38. Shedden PM, Wiley MJ : Early stages of development in the caudal neural tube of the Golden Syrian hamster (Mesocricetus auratus). Anat Rec $219: 180-185,1987$

39. Shih J, Fraser SE : Characterizing the zebrafish organizer: microsurgical analysis at the early-shield stage. Development 122 : 1313-1322, 1996

40. Shimokita E, Takahashi Y : Secondary neurulation: fate-mapping and gene manipulation of the neural tube in tail bud. Dev Growth Differ 53 : 401-410, 2011

41. Taniguchi Y, Kurth T, Weiche S, Reichelt S, Tazaki A, Perike S, et al. : The posterior neural plate in axolotl gives rise to neural tube or turns anteriorly to form somites of the tail and posterior trunk. Dev Biol 422 : 155170, 2017

42. Tucker AS, Slack JM : The Xenopus laevis tail-forming region. Development $121: 249-262,1995$ 
43. Wilson V, Beddington RS : Cell fate and morphogenetic movement in the late mouse primitive streak. Mech Dev 55 : 79-89, 1996

44. Wilson V, Olivera-Martinez I, Storey KG : Stem cells, signals and vertebrate body axis extension. Development 136 : 1591-1604, 2009

45. Yang HJ, Lee DH, Lee YJ, Chi JG, Lee JY, Phi JH, et al. : Secondary neuru- lation of human embryos: morphological changes and the expression of neuronal antigens. Childs Nerv Syst 30 : 73-82, 2014

46. Zwilling $\mathrm{E}$ : Restitution of the tail in the early chick embryo. J Exp Zool $91:$ 453-463, 1942 